\title{
Atrial fibrillation mimicking ventricular fibrillation confuses an automated external defibrillator
}

\author{
M. Hulleman ${ }^{1}$ (D) $\cdot$ M. T. Blom ${ }^{1} \cdot$ A. Bardai ${ }^{1,2} \cdot$ H. L. Tan ${ }^{1} \cdot$ R. W. Koster ${ }^{1}$ \\ Published online: 12 March 2018 \\ (c) The Author(s) 2018. This article is an open access publication.
}

A 47-year-old man suffered an out-of-hospital cardiac arrest. For scientific purposes, we analysed the electrocardiogram of the deployed automated external defibrillator (AED) [1]. Initially, the AED detected a non-shockable rhythm, caused by atrial fibrillation (AF) with high-degree atrioventricular block and slow ventricular escape rhythm (Fig. 1 panel A). During continued rhythm analysis, however, no escape beats occurred and a shock was delivered because isolated AF waves were erroneously interpreted as ventricular fibrillation (VF) (Fig. 1 panels B-C). In-hospital AF mimicking VF in monitored patients has been described before [2, 3], but ours is the first report of inappropriate AED therapy for AF mimicking VF during out-of-hospital cardiac arrest. The sensitivity and specificity of AED algorithms is related to the defibrillation threshold as defined in the AED algorithm [4]. The defibrillation threshold in the AED in this case report was $0.08 \mathrm{mV}$, which is lower than other AEDs where it ranges from 0.1 to $0.2 \mathrm{mV}$. An amplitude below the threshold is considered asystole. In this case the AF waves were between 0.08 and $0.2 \mathrm{mV}$, interpreted as 'fine' VF.

It is important to recognise that in current clinical practice we do not routinely retrieve and analyse AED electrocardiograms, and AED shocks are generally used as a proxy for VF. This practice may result in treatment errors. Clearly, AED electrocardiograms should always be analysed to allow for correct clinical decision-making.

M. Hulleman

m.hulleman@amc.nl

1 Department of Cardiology, Academic Medical Center-Heart Center, Amsterdam, The Netherlands

2 Department of Clinical Genetics, Academic Medical Center, Amsterdam, The Netherlands
Acknowledgements We are grateful for the cooperation of the Airport Medical Services in the recording of this case.

Funding M. Hulleman is supported by a grant from the Netherlands Heart Foundation (grant 2013T034). M.T.B. and H.L.T. are supported through ESCAPE-NET, a project that has received funding from the European Union's Horizon 2020 research and innovation programme under grant agreement No 733381. R.W. Koster is supported by a significant unconditional grant of Physio Control Inc, Redmond WA for the data collection in the ARREST studies.

Conflict of interest M. Hulleman, M.T. Blom, A. Bardai, H.L. Tan and and R.W. Koster declare that they have no competing interests.

Open Access This article is distributed under the terms of the Creative Commons Attribution 4.0 International License (http:// creativecommons.org/licenses/by/4.0/), which permits unrestricted use, distribution, and reproduction in any medium, provided you give appropriate credit to the original author(s) and the source, provide a link to the Creative Commons license, and indicate if changes were made.

\section{References}

1. Blom MT, van Hoeijen DA, Bardai A, et al. Genetic, clinical and pharmacological determinants of out-of-hospital cardiac arrest: rationale and outline of the AmsteRdam Resuscitation Studies (ARREST) registry. Open Heart. 2014;1:e112.

2. Ornato JP, Gonzales ER, Garnett R. Atrial fibrillation with high degree atrioventricular block masquerading as ventricular fibrillation masquerading as asystole during cardiac arrest. Crit Care Med. 1987;15:334-5.

3. Katz A, Snir Y, Wagshal AB, et al. Pseudoventricular fibrillation. Crit Care Med. 2001;29:1835-6.

4. Israelsson J, von Wangenheim B, Arestedt K, et al. Sensitivity and specificity of two different automated external defibrillators. Resuscitation. 2017;120:108-12. 
Fig. 1 Electrocardiogram of automated external defibrillator showing initial rhythm assessment (panel 1), and second rhythm check after two minutes (panel 2) resulting in a shock (panel 3)

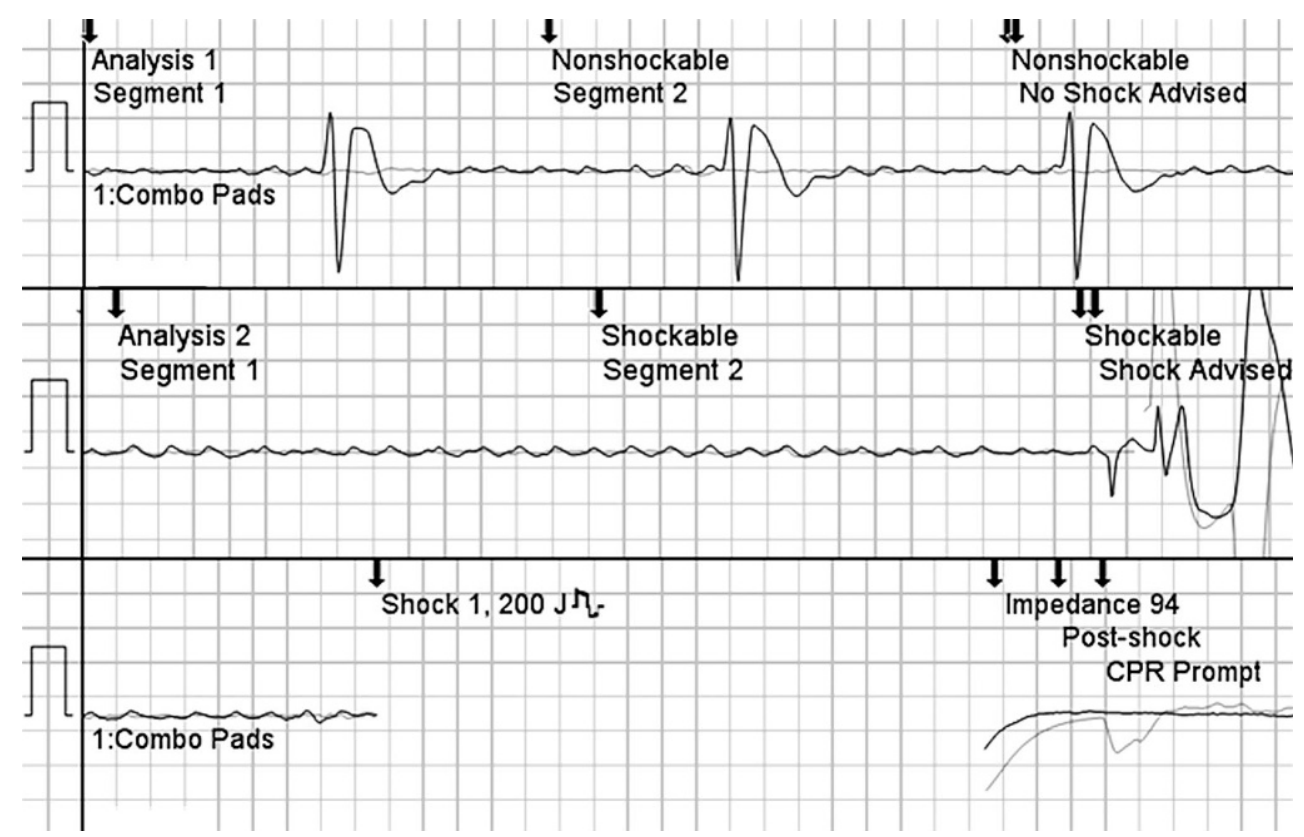

